\title{
PROPRIEDADES FÍSICAS DE CHAPAS DE PARTÍCULAS AGLOMERADAS FABRICADAS COM RESÍDUOS DO PROCESSAMENTO MECÂNICO DA MADEIRA DE Pinus elliotii Engelm.
}

\author{
DIMENSIONAL STABILITY OF PARTICLEBOARD MANUFACTURED WITH WOOD \\ MECHANICAL PROCESSING RESIDUES OF SLASH PINE
}

Lourdes Patricia Elias Dacosta ${ }^{1}$ Clovis Roberto Haselein ${ }^{2}$ Elio José Santini ${ }^{3}$ Paulo Renato Schneider ${ }^{4}$ Leandro Calegari ${ }^{5}$

\begin{abstract}
RESUMO
Este trabalho foi realizado com o objetivo de avaliar a estabilidade dimensional de painéis aglomerados confeccionados com resíduos de serraria e marcenaria, resultantes do processamento mecânico da madeira Pinus elliottii Engelm. Chapas com densidades nominais de 0,6 e $0,7 \mathrm{~g} / \mathrm{cm}^{3}$ foram produzidas com dois tipos de resíduos, cavacos e maravalhas, utilizados puros ou misturados. O adesivo empregado na confecção das chapas foi a base de uréia-formaldeído em proporções de 4,8 e $12 \%$ com base no peso seco das partículas de madeira. As propriedades físicas avaliadas foram absorção d'água e inchamento em espessura. Os resultados evidenciaram que as chapas manufaturadas com partículas do tipo maravalha, que foram coladas com as proporções de 8 e $12 \%$ de adesivo, apresentaram qualidade satisfatória.
\end{abstract}

Palavras-chave: chapas aglomeradas; uréia-formaldeído; resíduos de madeira, Pinus elliottii.

\begin{abstract}
The objective of this research was the investigation of dimensional stability of particleboard made with slash pine wood residues. The boards were manufactured with chips and shaves, resulting from sawmill and planermill industries, respectively, used pure or mixed. The adhesive used was urea-formaldehyde in proportions of 4,8 and $12 \%$, based on the ovendry weight of the particles. Board density investigated were 0.6 and $0.7 \mathrm{~g} / \mathrm{cm}^{3}$. The properties evaluated were water absorption and thickness swelling. The best boards were those made of shaves glued with 8 and $12 \%$ adhesive content.
\end{abstract}

Key words: particleboard; urea-formaldehyde; wood residues, slash pine.

\section{INTRODUÇÃO}

Em principio, todo e qualquer material lignocelulósico pode ser utilizado como matéria-prima para a fabricação de chapas de partículas. Entretanto, só as madeiras de folhosas e de coníferas se apresentam como fonte permanente e ininterrupta de elementos lignocelulósicos para a produção desses produtos. No Brasil, a madeira utilizada para a produção de painéis aglomerados provém, em sua totalidade, de florestas plantadas.

Fagundes (2003) comenta que a probabilidade da utilização de sobras e resíduos de madeira na fabricação de chapas de madeira reconstituída serve para aumentar a sustentabilidade na utilização das florestas, especialmente das plantadas, como insumo na produção madeirável.

Sabe-se que a indústria de chapas de madeira aglomerada utiliza grandes quantidades de madeira roliça e, em menor extensão, resíduos produzidos por outras indústrias madeireiras.

É importante, então, otimizar a utilização desses resíduos madeiráveis como fonte de matéria-prima para a indústria de chapas de madeira aglomerada, logrando assim a sustentabilidade dos benefícios sociais, econômicos e empresariais.

1. Engenheira Florestal, Doutoranda pelo Programa de Pós-Graduação em Engenharia Florestal, Centro de Ciências Rurais, Universidade Federal de Santa Maria, CEP 97105-900, Santa Maria (RS). lpatricia@mail.ufsm.br

2. Engenheiro Florestal, PhD, Professor Adjunto do Departamento de Ciências Florestais, Centro de Ciências Rurais, Universidade Federal de Santa Maria, CEP 97105-900, Santa Maria (RS). haseleic@ccr.ufsm.br

3. Engenheiro Florestal, Dr., Professor Adjunto do Departamento de Ciências Florestais, Centro de Ciências Rurais, Universidade Federal de Santa Maria, CEP 97105-900, Santa Maria (RS). santinie@ccr.ufsm.br

4. Engenheiro Florestal, Dr., Professor Titular do Departamento de Ciências Florestais, Centro de Ciências Rurais, Universidade Federal de Santa Maria, CEP 97105-900, Santa Maria (RS). paulors@ccr.ufsm.br

5. Engenheiro Florestal, Mestrando pelo Programa de Pós-Graduação em Engenharia Florestal, Centro de Ciências Rurais, Universidade Federal de Santa Maria, CEP 97105-900, Santa Maria (RS). leandrocalegari@yahoo.com.br

Recebido para publicação em 20/10/2004 e aceito em 27/09/2005. 
Haselein et al. (2002) comenta que o termo partícula é definido no padrão ASTM D1554 (1999) como o componente agregado de uma chapa de partículas de madeira ou outro material lignocelulósico, incluindo todas as menores subdivisões da madeira, fabricadas por meios mecânicos.

Dessa forma, a resistência mecânica das partículas obtidas é consideravelmente mais baixa do que a da madeira que as originaram. Ou melhor, a estrutura final do painel representa uma unidade que possui vazios e rupturas, que reduzem a resistência mecânica, em vez de um elemento maciço.

Segundo Peixoto e Brito (2000) e Haselein et al. (2002), partículas geradas por moinhos de martelos podem ser produzidas partindo de resíduos que vêm da industrialização da madeira.

Vignote e Jiménez (1996) afirmam que, ao classificar as partículas, deve-se ter em consideração que partículas muito pequenas ampliam a área de superfície, e, assim, as exigências de resina aumentam.

Madeira de alta densidade produz menor área de partículas, que levaria a uma redução na quantidade de adesivo aplicada. Pode ocorrer também menor área de contato entre as partículas, por causa do menor número destas para um mesmo peso, o que leva à formação de chapas com baixa resistência mecânica. Assim, deve-se aumentar a densidade da chapa ou a quantidade aplicada de adesivo, fazendo com que a esperada economia de adesivo desapareça (Roque, 1998).

A pesquisa realizada por Brito (1995) demonstrou a viabilidade de utilização de partículas de madeira com $9 \%$ de teor de umidade na manufatura de chapas de aglomerados partindo maravalhas de Pinus elliottii Engelm.

Com relação às propriedades físicas, a variação nas dimensões da chapa de partículas ocorre sobretudo em relação à sua espessura (Vignote e Jiménez, 1996).

Segundo Iwakiri et al. (1999), uma limitação da chapa de madeira aglomerada produzida no Brasil é a estabilidade dimensional, sendo esta influenciada pelas pequenas dimensões das partículas utilizadas.

$\mathrm{O}$ inchamento em espessura constitui-se em uma das propriedades mais importantes em termos de estabilidade dimensional em painéis. Essa propriedade pode ser afetada pela espécie da madeira, geometria das partículas, densidade dos painéis, teor de resina, teor de parafina, eficiência na aplicação da cola e condições de prensagem (Brito, 1995).

Iwakiri et al. (1996), em seu estudo sobre produção de aglomerados com mistura de Pinus elliottii e Eucalyptus dunnii, demonstrou que, com o aumento na razão de compactação, pela maior proporção de pinus no painel, aumentam os valores médios de absorção de água e de inchamento em espessura.

Hillig (2000) observou que, na confecção de chapas aglomeradas estruturais, utilizando partículas de Pinus elliottii Engelm. com várias dimensões nominais e densidade de $0,7 \mathrm{~g} / \mathrm{cm}^{3}$, e usando $8 \%$ de adesivo, o inchamento em espessura aumentou com o aumento da espessura das partículas.

Brito e Silva (2002), em seu estudo sobre as propriedades de chapas de partículas produzidas com Gmelina arborea Linn. Roxb. e Samanea saman (Jacq.) Merr., observaram que as chapas produzidas com partículas menores da primeira espécie mostraram os melhores resultados em inchamento em espessura.

Quanto ao efeito da quantidade de resina aplicada nas partículas, pode-se observar o resultado obtido por Albuquerque (2002), no qual um aumento de 8 para 12\%, no teor de adesivo, causou uma diminuição na absorção d'água bem como no inchamento em espessura.

A densidade da chapa está intimamente associada à densidade das madeiras empregadas na sua fabricação e à taxa de compressão necessária para a sua compactação (Hillig, 2000).

Vignote e Jiménez (1996) comentam que, na parte externa, a densidade da chapa pode chegar a 1,2 $\mathrm{g} / \mathrm{cm}^{3}$ e, na parte interna, pode ser de $0,5 \mathrm{~g} / \mathrm{cm}^{3}$. Essa heterogeneidade pode fazer com que a chapa não permita perfis e acabamentos de boa qualidade.

Vital e Wilson (1980) afirmam que o efeito da densidade da chapa no inchamento em espessura deve-se mais à umidade relativa de exposição. Em condições de baixa umidade relativa, o inchamento em espessura independe da densidade, enquanto que em exposição a altas umidades relativas aumenta com o aumento da densidade. 
Usando-se partículas grossas, em painéis menos densos, ocorre uma maior absorção de água, e, em painéis mais densos, um maior inchamento em espessura (Albuquerque, 2002).

Baseando-se no exposto, o presente estudo foi realizado com o objetivo de avaliar as propriedades físicas dos painéis aglomerados confeccionados com resíduos de pinus resultantes do processamento mecânico da madeira.

\section{MATERIAL E MÉTODOS}

\section{Fabricação das chapas}

A matéria-prima utilizada corresponde a resíduos de processamento mecânico da madeira de Pinus elliottii Engelm., obtidos por ocasião do desdobro na serraria e do beneficiamento na marcenaria da empresa Todesmade, situada no município de Cachoeira do Sul (RS). A empresa utiliza serra de fita dupla tipo tanden e serra circular múltipla para o desdobro primário; o processamento secundário é realizado por meio de serras de fita simples e serras circulares múltiplas. Os equipamentos de beneficiamento são constituídos sobretudo por plainas, com diversas características em razão do produto final, e serras circulares destopadeiras.

Os resíduos do processamento mecânico da madeira, constituídos de serragem, casca, costaneiras, destopos e refilos, foram reprocessados em um picador pela própria empresa. No momento da coleta, o resíduo estava constituído por cavacos, serragem e casca, numa proporção de 55,6, 22,7 e 21,7\% respectivamente. Já as maravalhas, resultantes do beneficiamento, não foram reprocessadas.

No Laboratório de Produtos Florestais da UFSM, os cavacos misturados com casca e serragem foram reduzidos a partículas com o uso de um moinho de martelos. Os cavacos (denominação dada à mistura de cavacos, serragem e casca que foi processada em moinho de martelos) e as maravalhas foram colocados em estufa com ventilação forçada, a $60^{\circ} \mathrm{C}$, até atingir um teor de umidade de aproximadamente $3 \%$. Esse teor de umidade corresponde ao valor comumente empregado industrialmente na fabricação desse tipo de chapa.

Após a secagem, o material (cavacos e maravalhas) foi peneirado para remoção dos finos (usando-se peneira com malhas de $1,53 \times 1,90 \mathrm{~mm}$ ) e das partículas muito grandes (ficaram retidas em peneira com malhas de 3,3 x 2,6 mm). As partículas selecionadas foram armazenadas em sacos de polietileno até o momento da fabricação das chapas.

$\mathrm{O}$ adesivo empregado foi à base de uréia-formaldeído. Para determinar o teor de sólidos da resina de uréia formaldeído e da parafina, aplicou-se o procedimento da norma ASTM D 1490-96 (1999).

Foram confeccionadas 54 chapas aglomeradas, com dimensões de 50 x 50 x 0,95 cm. As variáveis de estudo para cada tratamento foram a proporção de cavacos ou maravalhas utilizadas $(0,50$ e $100 \%)$, a densidade da chapa $\left(0,6\right.$ e $\left.0,7 \mathrm{~g} / \mathrm{cm}^{3}\right)$ e o teor de adesivo $(4,8$ e $12 \%)$.

A prensagem à quente foi realizada em prensa de laboratório marca OMECO, acionada por um pistão hidráulico com capacidade de 100 toneladas de aplicação de carga, e pratos com dimensões de 60 x $60 \mathrm{~cm}$. A pressão específica aplicada foi de $30 \mathrm{kgf} / \mathrm{cm}^{2}$, e a temperatura dos pratos foi ajustada em $180^{\circ} \mathrm{C}$. O tempo de fechamento da prensa foi de 40 segundos, e o tempo total de prensagem foi de 8 minutos.

Depois da prensagem, as chapas foram identificadas, esquadrejadas para a confecção das chapas e conduzidas à câmara climática para serem condicionadas à temperatura de $20^{\circ} \mathrm{C}$ e umidade relativa de $65 \%$.

\section{Testes físicos}

Foram avaliadas, pela norma norte-americana D-1037-93 (ASTM, 1995), as seguintes propriedades das chapas: inchamento em espessura após 2 (IE 2) e 24 horas (IE 24), e absorção d'água em peso após 2 $(\mathrm{Ab} 2)$ e 24 horas ( $\mathrm{Ab} 24)$ de imersão em água.

Nesses ensaios, foram utilizados corpos de prova de $15 \times 15 \mathrm{~cm}$, lixados e submersos em água à temperatura de $20^{\circ} \mathrm{C}$. O material foi colocado na posição horizontal e mantido submerso a uma profundidade de $25 \mathrm{~mm}$, por meio de pesos em forma de grade.

Foram determinados a espessura média e o peso das peças condicionadas na câmara climática após 2 e 24 horas de submersão na água. Para medição da espessura, foi utilizado um relógio comparador, adaptado 
especialmente para tal finalidade. As medidas de comprimento e largura foram realizadas com paquímetro, e a massa foi determinada com balança analítica eletrônica.

Após as medições, os corpos de prova foram levados à estufa a $103^{\circ} \mathrm{C}$, para a determinação do teor de umidade base seca, no momento de cada medição. Assim, foram determinados os teores de umidade dos corpos de prova à umidade de equilíbrio na câmara climatizada (Tue), após 2 horas (Tu 2) e 24 horas (Tu 24) de imersão em água.

\section{Modelo experimental e análise dos resultados}

O modelo experimental utilizado foi o delineamento inteiramente casualizado, com três repetições, segundo um modelo trifatorial ( $3 \times 3 \times 2)$, no qual os tratamentos avaliados mostram as combinações empregadas para a confecção das chapas de partículas de cavacos e maravalhas, com o adesivo e a densidade conforme pode ser visto na Tabela 1 .

De modo a facilitar os cálculos estatísticos, foram utilizados, no caso do material empregado, valores de 100,50 e $0 \%$ para indicar a presença de resíduos de serraria (cavacos) em cada chapa. Destes percentuais, $100 \%$ indica uma chapa feita só com esse material, $50 \%$ a mistura de cavacos e maravalha em partes iguais, e $0 \%$ significa que a chapa é feita só com maravalha.

TABELA 1: Tratamentos aplicados no estudo dos painéis aglomerados, fabricados com resíduos de processamento mecânico.

TABLE 1: List of treatments used for the manufacturing of the particleboard from wood mechanical processing residues.

\begin{tabular}{clc|c}
\hline Tratamento & \multicolumn{1}{c}{ Tipo de resíduo } & Teor de adesivo $(\%)$ & Densidade $\left(\mathrm{g} / \mathrm{cm}^{3}\right)$ \\
\hline 1 & Cavacos & 4 & 0,60 \\
2 & Cavacos & 8 & 0,60 \\
3 & Cavacos & 12 & 0,60 \\
4 & Cavacos & 4 & 0,70 \\
5 & Cavacos & 8 & 0,70 \\
6 & Cavacos & 12 & 0,70 \\
7 & Maravalha & 4 & 0,60 \\
8 & Maravalha & 8 & 0,60 \\
9 & Maravalha & 12 & 0,60 \\
10 & Maravalha & 4 & 0,70 \\
11 & Maravalha & 8 & 0,70 \\
12 & Maravalha & 12 & 0,70 \\
13 & Cavacos e Maravalha & 4 & 0,60 \\
14 & Cavacos e Maravalha & 8 & 0,60 \\
15 & Cavacos e Maravalha & 12 & 0,60 \\
16 & Cavacos e Maravalha & 4 & 0,70 \\
17 & Cavacos e Maravalha & 8 & 0,70 \\
18 & Cavacos e Maravalha & 12 & 0,70 \\
\hline
\end{tabular}

Em que: cavacos = denominação atribuída à mistura dos resíduos do desdobro da madeira; maravalha = denominação atribuída aos resíduos do beneficiamento da madeira.

Após a realização dos ensaios físicos, os resultados obtidos foram analisados pela correlação de médias e análise de regressão, relacionando-se os resultados dos testes com as variáveis do estudo, e adotando-se níveis de significância de $5 \%$ de probabilidade.

\section{RESULTADOS E DISCUSSÃO}

\section{Absorção d'água}

Os valores médios de absorção d'água e inchamento em espessura, após 2 e 24 horas de imersão, são apresentados na Tabela 2. Observa-se que os valores médios de absorção de água oscilaram entre 10,95 a $34,90 \%$ e 47,7 a $91,96 \%$ para 2 e 24 horas de imersão respectivamente. Os valores médios de inchamento em espessura variaram entre 2,94 a $11,06 \%$ e $13,36 \%$ a $41,39 \%$ para 2 e 24 horas de imersão respectivamente. 
TABELA 2: Valores médios de absorção de água e inchamento em espessura, após 2 e 24 horas de imersão em água encontrados para os diferentes tratamentos.

TABLE 2: Average values of water absorption and thickness swelling after 2 and 24 hours of water soaking for the different treatments.

\begin{tabular}{c|c|c|c|c|c|c|c|c|c}
\hline Trat. & $\begin{array}{c}\text { Cavacos } \\
(\%)\end{array}$ & $\begin{array}{c}\text { Maravalha } \\
(\%)\end{array}$ & $\begin{array}{c}\text { Ta } \\
(\%)\end{array}$ & $\begin{array}{c}\text { Dn } \\
\left(\mathrm{g} / \mathrm{cm}^{3}\right)\end{array}$ & $\begin{array}{c}\text { Do } \\
\left(\mathrm{g} / \mathrm{cm}^{3}\right)\end{array}$ & $\begin{array}{c}\text { Ab2 } \\
(\%)\end{array}$ & $\begin{array}{c}\text { Ab24 } \\
(\%)\end{array}$ & $\begin{array}{c}\text { IE2 } \\
(\%)\end{array}$ & $\begin{array}{c}\text { IE24 } \\
(\%)\end{array}$ \\
\hline 1 & 100 & 0 & 4 & 0,60 & 0,54 & 22,50 & 84,20 & 8,71 & 35,49 \\
2 & 100 & 0 & 8 & 0,60 & 0,58 & 22,14 & 90,85 & 7,00 & 25,75 \\
3 & 100 & 0 & 12 & 0,60 & 0,56 & 34,90 & 75,67 & 8,52 & 23,50 \\
4 & 100 & 0 & 4 & 0,70 & 0,65 & 19,99 & 84,08 & 8,39 & 40,04 \\
5 & 100 & 0 & 8 & 0,70 & 0,60 & 34,56 & 90,33 & 11,06 & 28,67 \\
6 & 100 & 0 & 12 & 0,70 & 0,60 & 29,11 & 76,54 & 8,77 & 30,14 \\
7 & 0 & 100 & 4 & 0,60 & 0,57 & 15,60 & 80,68 & 3,69 & 21,99 \\
8 & 0 & 100 & 8 & 0,60 & 0,57 & 17,16 & 64,56 & 3,16 & 14,55 \\
9 & 0 & 100 & 12 & 0,60 & 0,59 & 13,70 & 53,26 & 2,99 & 13,36 \\
10 & 0 & 100 & 4 & 0,70 & 0,65 & 17,11 & 86,22 & 5,11 & 32,59 \\
11 & 0 & 100 & 8 & 0,70 & 0,65 & 11,70 & 58,93 & 3,71 & 21,49 \\
12 & 0 & 100 & 12 & 0,70 & 0,66 & 10,95 & 47,70 & 2,94 & 14,20 \\
13 & 50 & 50 & 4 & 0,60 & 0,56 & 18,74 & 88,61 & 7,92 & 36,73 \\
14 & 50 & 50 & 8 & 0,60 & 0,57 & 17,69 & 74,63 & 7,14 & 26,88 \\
15 & 50 & 50 & 12 & 0,60 & 0,59 & 19,33 & 66,44 & 4,67 & 19,39 \\
16 & 50 & 50 & 4 & 0,70 & 0,64 & 20,74 & 91,96 & 6,36 & 41,39 \\
17 & 50 & 50 & 8 & 0,70 & 0,66 & 15,50 & 78,73 & 4,55 & 30,24 \\
18 & 50 & 50 & 12 & 0,70 & 0,64 & 17,17 & 56,63 & 5,25 & 20,43 \\
\hline
\end{tabular}

Em que: Trat $=$ tratamentos; $\mathrm{Ta}=$ teor de adesivo $(\%) ; \mathrm{Dn}=$ densidade nominal $\left(\mathrm{g} / \mathrm{cm}^{3}\right) ;$ Do $=$ densidade observada $\left(\mathrm{g} / \mathrm{cm}^{3}\right) ; \mathrm{Ab} 2=$ absorção em peso ( 2 horas de imersão); Ab24 = absorção em peso (24 horas de imersão); IE2 = inchamento em espessura ( 2 horas de imersão); IE24 = inchamento em espessura (24 horas de imersão).

A correlação entre as variáveis evidenciou que, após 24 horas, tanto a absorção de água como o inchamento em espessura, apresentaram valores significativos em todas as variáveis independentes analisadas.

Em relação aos cavacos, conforme sua proporção na mistura foi aumentada a absorção, e o inchamento d'água também aumentou. Quanto ao teor de adesivo, a correlação indica que com o aumento do teor de adesivo, ocorre uma redução na absorção e no inchamento. No que se refere à densidade da chapa, verificou-se que aumentando seus valores, a absorção diminui e o inchamento aumenta (Tabela 3).

TABELA 3: Correlação de Pearson entre as propriedades de estabilidade dimensional e as variáveis de fabricação das chapas.

TABLE 3: Pearson correlation between dimensional stability and the manufacturing variables.

\begin{tabular}{l|c|c}
\hline Variáveis de fabricação & $\mathrm{Ab} \mathrm{24}(\%)$ & $\mathrm{IE} \mathrm{24}(\%)$ \\
\hline Proporção de serragem (\%) & $0,45238(* *)$ & $0,47723(* *)$ \\
Teor de adesivo $(\%)$ & $-0,50986(* *)$ & $-0,63593(* *)$ \\
Densidade observada $\left(\mathrm{g} / \mathrm{cm}^{3}\right)$ & $-0,20598(*)$ & $0,29562(* *)$ \\
\hline
\end{tabular}

Em que: AB 24 = absorção d'água 24 horas; IE 24 = inchamento em espessura após 24 horas de imersão; * = correlação significativa a $5 \% ; * *=$ correlação significativa a $1 \%$.

As Figuras 1 e 2 mostram os gráficos com o modelo de regressão, para absorção de água após 24 horas e inchamento em espessura após 24 horas, com valores respectivamente ajustados em razão da densidade, para as três proporções de cavacos e mantendo fixa a percentagem de adesivo cujos coeficientes e precisões se encontram na Tabela 4. 


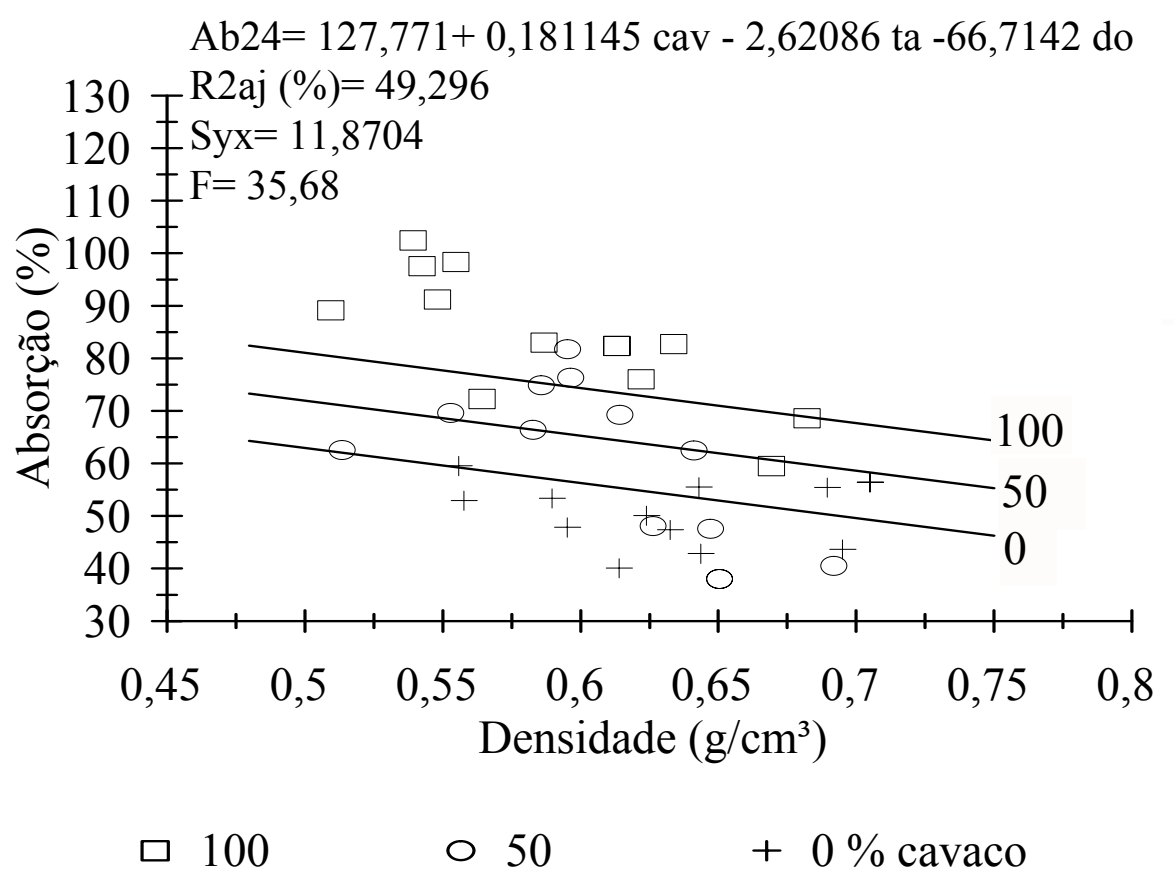

FIGURA 1: Valores de absorção de água após 24 horas de imersão (Ab24), observados e ajustados em função da densidade (do), da proporção de cavacos e teor de adesivo (ta) de $12 \%$.

FIGURE 1: Water absorption after 24 hours soaking observed and adjusted as a function of board density (do), chip proportion and adhesive content (ta) of $12 \%$.

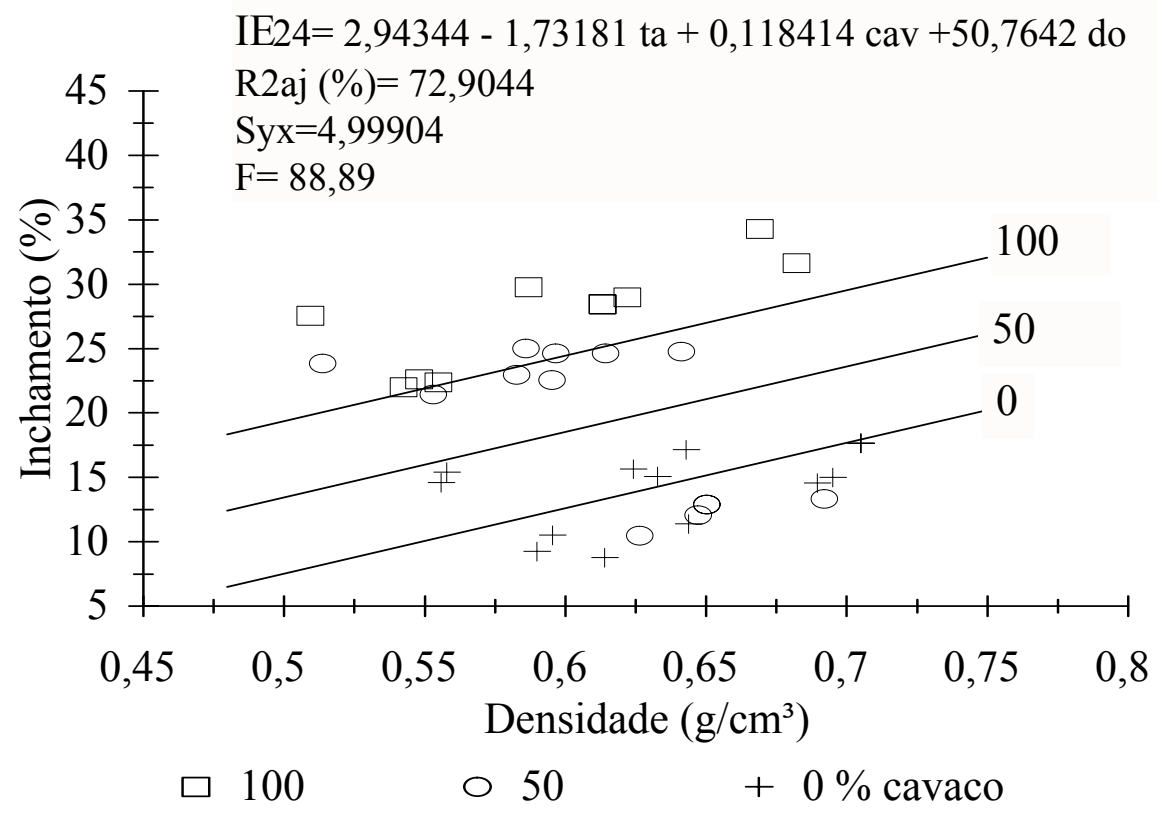

FIGURA 2: Valores de inchamento em espessura 24 horas (IE24) observados e ajustados em função da densidade (do), a proporção de cavacos (cav) e teor de adesivo (ta) de $12 \%$.

FIGURE 2: Thickness swelling after 24 hours soaking observed and adjusted as a function of board density, chip proportion and adhesive content. 
Segundo o modelo matemático ajustado, pode-se verificar uma tendência de diminuição da absorção com o aumento do teor de adesivo e da densidade na chapa.

TABELA 4: Equações ajustadas para as propriedades das chapas em função das variáveis de fabricação. TABLE 4: Fitted equations for the properties of the board as a function of the manufacturing parameters.

\begin{tabular}{l|c|c|c|c|c}
\hline Propriedade & Equação ajustada & $\mathrm{F}$ & Syx & $\mathrm{R}^{2}$ aj. (\%) & DU \\
\hline $\mathrm{Abs}_{24}$ & $127,771+0,181145 \mathrm{cav}-2,62086 \mathrm{ta}-66,7142$ do & 35,68 & 11,87 & 49,29 & 1,232 \\
$\mathrm{IE}_{24}$ & $2,94344-1,73181 \mathrm{ta}+0,118414 \mathrm{cav}+50,7642$ do & 88,89 & 4,99 & 72,90 & 1,032 \\
\hline
\end{tabular}

Em que: ta = teor de adesivo (\%); do = densidade observada $\left(\mathrm{g} / \mathrm{cm}^{3}\right) ;$ cav = a proporção de cavacos; $\mathrm{Ab}_{24}=$ absorção em peso após 24 horas de imersão (\%); $\mathrm{IE}_{24}=$ inchamento em espessura após 24 horas de imersão (\%); $\mathrm{F}=$ teste de $\mathrm{F}$; Syx = erro padrão da estimativa; $\mathrm{R}^{2}$ aj. = coeficiente de determinação ajustado; $\mathrm{DU}=$ teste de Durbin-Watson.

$\mathrm{Na}$ análise de absorção de água em função das variáveis de produção, o melhor modelo para explicar a absorção em peso após 24 horas, para todas as chapas fabricadas, inclui as proporções de cavacos, teor de adesivo e densidade (valor de $\mathrm{P}$ menor que 0,01 ).

Os valores mais baixos de inchamento em espessura, independentemente das densidades, foram obtidos com o uso de adesivo em proporção de 12\%. Isto foi verificado também por Albuquerque (2002), que observou uma diminuição em absorção de água e inchamento em espessura, aumentando o teor de adesivo de 8 para $12 \%$.

Foi observado que os corpos de prova de maravalha apresentaram menor inchamento em espessura, sendo que os tratamentos 8,9 e 12 produziram chapas que atenderam às exigências da norma DIN (1971), sendo o valor máximo admitido de $15 \%$ para IE $24 \mathrm{~h}$.

Sobre isso, Maciel (2001) observou que as chapas elaboradas com madeira de Pinus são menos sensíveis ao inchamento em espessura do que aquelas produzidas com madeira de eucalipto.

Iwakiri et al. (1996), estudando a mistura de espécies de Eucalyptus dunnii e Pinus elliottii, observaram que, com o aumento da proporção de Pinus no painel, se elevavam os valores médios de absorção de água e inchamento em espessura.

O mesmo fato foi observado por Hillig (2000) ao usar proporções de Pinus na fabricação de chapas, o que influenciou no inchamento em espessura comparado com eucaliptus e acácia, atingindo uma média de $25,31 \%$.

O melhor modelo encontrado para explicar o inchamento em espessura inclui proporção de cavacos, teor de adesivo e densidade que são significativos no modelo (valor de P inferior a 0,01). Deve-se salientar o efeito marcante da proporção de cavacos, provavelmente relacionada à presença de casca e do adesivo no inchamento em espessura. Além disso, apesar da densidade da chapa causar um decréscimo na absorção de água, há um aumento do IE $24 \mathrm{~h}$ com o aumento da densidade. Isso pode ser explicado, em parte, pela liberação das tensões geradas durante a prensagem (spring back), que é acelerada pela absorção de água (Suchsland, 1973).

\section{CONCLUSÕES}

Com base nos resultados obtidos neste estudo, as seguintes conclusões são apresentadas:

No inchamento em espessura, não foram atingidos os valores requeridos na maioria dos tratamentos. Porém, mas pode o emprego de maravalhas, independentemente da densidade ou do teor de adesivo, resultou num menor inchamento quando comparado com o resíduo de serraria ou com a mistura de ambos.

A variável, que exerceu maior influência na absorção de água e no inchamento em espessura, foi o teor de adesivo, seguido da densidade, e da proporção e tipo de material. Constatou-se que aumentando a proporção de cavacos, a absorção e inchamento aumentaram.

O cavaco foi o material que apresentou os resultados menos satisfatórios nessas propriedades. 
Recomenda-se o estudo sobre a mistura desse tipo de resíduos, com outras espécies, ou outros tipos de partículas, considerando também a proporção de casca ideal a ser incluída se fossem utilizados na fabricação de chapas de três camadas e usados como miolo destas.

\section{AGRADECIMENTOS}

Os autores agradecem às empresas Todesmade de Cachoeira do Sul (RS) e Sinteko de Gravataí (RS), pelo fornecimento das partículas de madeira e do adesivo respectivamente.

\section{REFERÊNCIAS BIBLIOGRÁFICAS}

ALBUQUERQUE, C. E. C. de. Interações de variáveis no ciclo de prensagem de aglomerados. 2002. 150p. Tese (Doutorado em Ciências Florestais) - Universidade Federal de Paraná, Curitiba, 2002.

AMERICAN SOCIETY FOR TESTING AND MATERIALS. ASTM D1554. Standard termilogy relating to wood-base fiber and particle panel materials. Philadelphia, Pa. 1999.

AMERICAN SOCIETY FOR TESTING AND MATERIALS. Standard test methods for evaluating properties of wood-base fiber and particle panel materials, D 1037-93. Philadelphia, PA., 1995.

AMERICAN SOCIETY FOR TESTING AND MATERIALS. Standard specification for urea-formaldehyde resin adhesives, D 4690-95a. Philadelphia, PA., 1999.

BRITO, E. O. Produção de chapas de partículas de madeira a partir de maravalhas de Pinus elliottii Engelm. var. elliottii plantado no Sul de Brasil. 1995. 123p. Tese (Doutorado) - Universidade Federal do Paraná, Curitiba, 1995.

BRITO, E. O.; SILVA, V.C. Propriedade de chapas de partículas produzidas a partir de Gmelina arbórea Linn. Roxb. e Samanea saman ( Jacq.) Merr. Floresta e Ambiente, Rio de Janeiro, v. 9, n. 1, p. 127-134, 2002.

DIN. GERMAN STANDARDS COMMITTEE: Deutschen Normanausschuss. Specifications for particleboard. DIN 68761 (1) - 1961 (3). Holz, 1971.

FAGUNDES, H.A.V. Diagnóstico da produção de madeira serrada e geração de resíduos do processamento de madeira de florestas plantadas no Rio Grande do Sul. 2003. 180p. Dissertação (Mestrado em Engenharia Civil) Universidade Federal do Rio Grande do Sul, Porto Alegre, 2003.

HASELEIN, C. R. et al. Resistência mecânica e à umidade de painéis aglomerados com partículas de madeira de diferentes dimensões. Ciência Florestal, Santa Maria, v. 12, n. 2. p. 127-134, 2002.

HILLIG, E. Qualidade de chapas aglomeradas estruturais, fabricadas com madeiras de Pinus, Eucalipto e Acácia negra, puras ou misturadas, coladas com tanino- formaldeido. 2000. 96 p. Dissertação (Mestrado em Engenharia Florestal) - Universidade Federal de Santa Maria, Santa Maria, 2000.

IWAKIRI S. et al. Produção de chapas de partículas de madeira aglomerada de Pinus elliottii e Eucaliptus dunnii. Agrárias, Curitiba, v. 15, p. 33-41, 1996.

IWAKIRI S.; PEREIRA, S. J.; NISGOSKI, S. Influência da umidade, pressão e tempo de prensagem na produção de chapas de partículas de madeira aglomerada. Agrárias, Curitiba, v. 18. n. 1-2, p. 29-36, 1999.

MACIEL, A. da S. Chapas de partículas aglomeradas de madeira de Pinus elliottii ou Eucalyptus grandis, em mistura com poliestireno e polietileno tereftalato. 2001. 115p. Tese (Doutorado em Ciências Florestais) Universidade Federal de Viçosa, Viçosa, 2001.

PEIXOTO, G.L.; BRITO, E. O. Avaliação da granulométrica de partículas de Pinus taeda combinadas com adesivos comerciais para a fabricação de aglomerados. Revista Floresta e Ambiente, Rio de Janeiro, v. 7, n. 1, p. 60-67, 2000.

ROQUE, C. A. L. Painéis de madeira aglomerada. Disponível em:<www.bndes.gov.br>. Acesso em: 20 set. 1998.

SUCHSLAND, O. Higroscopic thickness swelling and related properties of selected commercial particleboard. Forest Products Journal, v. 23, n. 7, p. 26-30, 1973 
VIGNOTE P., S.; JIMÉNEZ P. F. J. Tecnología de la madera. Madrid : Ministerio de Agricultura, Pesca y Alimentación, 1996. 602p.

VITAL, B. R.; WILSON, J. B. Efeito da forma geométrica dos flocos e partículas, da densidade das chapas e do tipo de adesivo nas propriedades mecânicas das chapas de madeira aglomerada. Àrvore, Viçosa, v. 4, n. 2, p. 179-187, 1980. 